\author{
ALDONA SOPATA \\ Uniwersytet im. Adama Mickiewicza w Poznaniu \\ sopata@amu.edu.pl \\ ORCID: 0000-0002-0733-4455
}

\title{
Entwicklung der Informationsstruktur beim Erwerb des Deutschen als frühe Fremdsprache
}

\author{
Development of information structure \\ in acquisition of German \\ as an early foreign language
}

\begin{abstract}
The article investigates the use of pronouns to express subjects and objects in early acquisition of German as a foreign language. The main goal of the investigation is to shed some light on the process of development of information structure in the minds of young learners of a foreign language. The study investigates longitudinal data from children who were exposed to German in a school setting from the age of 7 or 9. The results of the empirical study show that children acquiring German in a school setting use a relatively high number of pronouns for subjects, but they rarely use pronouns to express objects. The pedagogical implication for developing an optimal outline for foreign language teaching in early childhood is the necessity to pay more attention to the development of pragmatic and discourse skills of children learning a foreign language.
\end{abstract}

KEYWORDS: pronouns, subject realisation, object realisation, German, acquisition, foreign language learning.

SCHLÜSSELWORTE: Pronomen, Subjektrealisierung, Objektrealisierung, Deutsch, Erwerb, Fremdsprachenlernen. 


\section{GLOTTODIDAKTISCHE REFLEXION ÜBER DIE ENTWICKLUNG DER KOGNITIVEN PROZESSE IN DER FREMDSPRACHE}

Das Forschungsziel der Glottodidaktik ist das Kennenlernen der fremdsprachlichen Lehr- und Lernprozesse und die Herausarbeitung optimaler Systeme des Lehrens und Lernens von Fremdsprachen. Die wichtigste Aufgabe der Glottodidaktik besteht daher darin, fremdsprachliche Lehr- und Lernprozesse $\mathrm{zu}$ beobachten, $\mathrm{zu}$ beschreiben, $\mathrm{zu}$ analysieren und zu erklären (vgl. Pfeiffer 2001a; 2001b). Glottodidaktische Prozesse umfassen mehrere Elemente, die als sog. Glottodidaktisches Gefüge (zum Begriff siehe Grucza 1978: 32) dargestellt werden: Lehrer, Lerner, die Sprache, die Methode des Lehrens und Lernens, die glottodidaktischen Materialien, die Unterrichtsbedingungen und die äußere Realität (vgl. Pfeiffer 2001a: 69). Pfeiffer (2001a: 75) unterstreicht, dass die primären Beziehungen in diesem Gefüge hochrelevant für das Funktionieren des Gefüges als Ganzes sind. Die sekundären Beziehungen des Gefüges treten erst dann auf, wenn ihr Einfluss auf andere Elemente relevant ist (Pfeiffer 2001a: 75).

Das Zusammenspiel der Elemente wird in der Abbildung 1 nach Pfeiffer (2001a; 2001b) gezeigt.

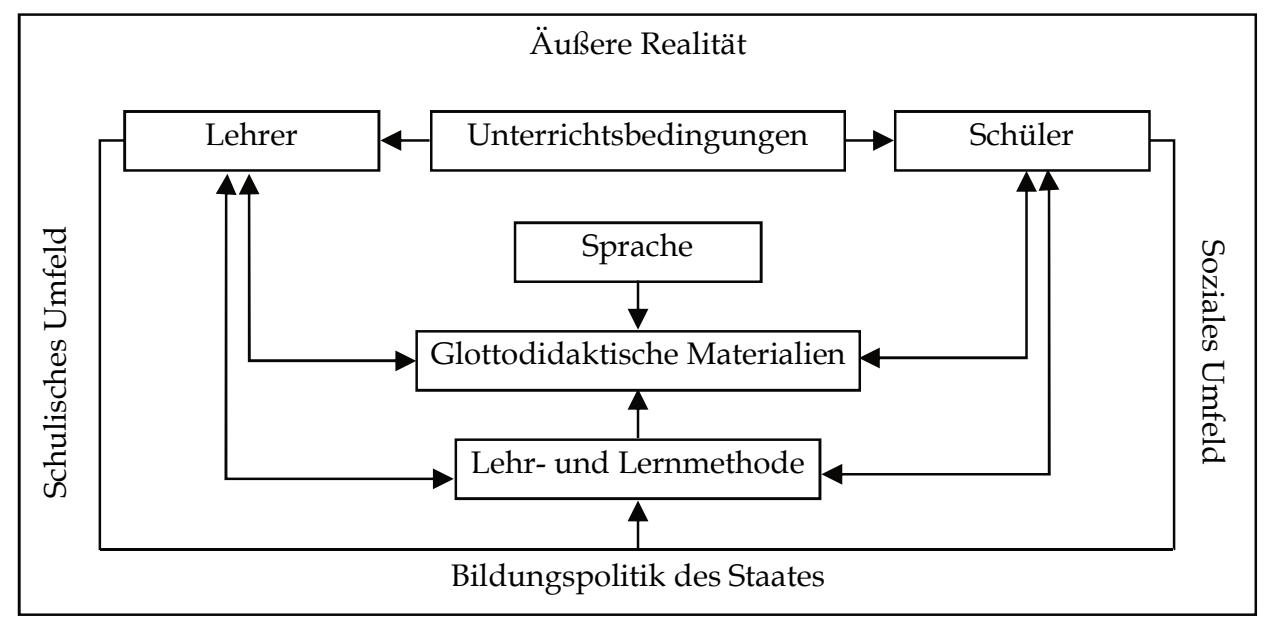

Abbildung 1. Das glottodidaktische Gefüge (Pfeiffer 2001a: 69).

Der Abbildung kann man entnehmen, dass alle Elemente des Gefüges invielgestaltigen Beziehungen zueinander stehen. Es ist ein offenes und dynamisches System (zu den Beziehungen zwischen unterschiedlichen Elementen des Systems vgl. Błażek 2007; Długosz 2017b; Skowronek 2013; 
Sopata 2004; 2010; 2017; Zuzok 2010). Im Zentrum des Gefüges stehen der Lerner und der Lehrer. Grucza (1983: 230) betont, dass der Forschungsgegenstand der Glottodidaktik der Mensch als Lerner und Vermittler sprachlicher Eigenschaften ist. Die Forschungsaufgabe der Glottodidaktik sieht Grucza (1993; 2007) daher in Erforschung der konstitutiven Eigenschaften, d.h. der Kenntnisse und Fähigkeiten der Sprachlerner und der Sprachlehrer, die ihren Handlungen zugrunde liegen. Die Theorie des Erwerbs einer Fremdsprache soll in erster Linie den Sprachlerner selbst und seine konstitutiven Eigenschaften rekonstruieren (vgl. Sadownik 1997: 14). Von der Fremdspracherwerbstheorie ist daher zu erwarten, dass sich mit ihrer Hilfe, Prognosen über den Sprachlerner, über den Ablauf der kognitiven Prozesse in seinem Kopf und über seine sprachliche Entwicklung erstellen lassen. Eine der grundlegenden Forschungsfragen der Glottodidaktik wurde von Grucza (2007: 22) folgenderweise formuliert: Worauf beruhen die Prozesse des Lehrens und Lernens von Fremdsprachen, angesichts dessen, dass die natürlichen Sprachen als Wissen (Elemente des Wissens) ihrer "Besitzer" aufzufassen sind und sie daher nicht (im wörtlichen Sinne) weitervermittelt werden können?

Die Verfolgung eines der Hauptziele der Glottodidaktik, d.h. Erhellung der Beschaffenheit der menschlichen Fähigkeit zum Lernen von Sprache und von Fremdsprache (vgl. Bielicka 2014; Długosz 2017a), ist im Fall von Kindern besonders interessant, weil die wesentlichen Bereiche des Erstspracherwerbs bei ihnen vor nicht allzu langer Zeit abgeschlossen worden sind. Pfeiffer (1980; 1995) erklärte eine komplexe Analyse der kognitiven Prozesse bei Kindern, die eine Fremdsprache erlernen, zu den primären Forschungsaufgaben der Glottodidaktik. Das Anliegen der vorliegenden Studie ist eben eine Einsicht in die kognitiven Prozesse beim Erwerb der Fremdsprache in der Kindheit.

\section{INFORMATIONSSTRUKTUR}

Die Erfassung von Informationen in einem Gespräch ist ein interaktiver Prozess, während dessen der Sprecher den Status des Wissens des Hörers beurteilen und berücksichtigen muss. Der Sprecher muss beispielsweise Annahmen darüber machen, welche Referenten für den Hörer neu, und welche bekannt sind, um für sie den besten Ausdruck auszuwählen.

Die mentale Repräsentation des ganzen Diskurses, d.h. der Diskursreferenten, der Informationen usw., wird in dem Begriff Informationsstruktur zusammengefasst (vgl. Zimmermann 2016). Unter diesem Begriff wird auch die Schnittstelle zwischen der Struktur und der Bedeutung von sprachlichen 
Äußerungen verstanden. An der Schnittstelle der mentalen Repräsentation des Diskurses spielen die Regeln des Strukturaufbaus, der Interpretation der Äußerungen und ihrer Verarbeitung mit den allgemeinen kognitiven Prozessen, wie Gedächtnis und Aufmerksamkeit zusammen (vgl. Lambrecht 1994). Die Informationsstruktur spielt eine determinierende Rolle in der Bindung von sprachlichen Äußerungen an die sich ständig ändernden kommunikativen Bedingungen und Kontexte (vgl. Zimmermann 2016). Sie zwingt den Gebrauch der entsprechenden Mittel oder Strukturen auf, durch die Informationen ausgedrückt werden. Die Informationsstruktur ist für den Wissenstransfer zwischen den Gesprächspartnern zuständig, der einerseits auf der ständigen Aktualisierung des mentalen Weltmodells des Gesprächspartners beruht und andererseits zum Aufbau des gemeinsamen Wissens durch den Äußerungsaustausch führt. Die Kategorien der Informationsstruktur, wie beispielsweise Fokus und Topik, sind universell und kommen in jeder natürlichen menschlichen Sprache vor. Wie sie sich jedoch in der sprachlichen Form niederschlagen, kann von Sprache zu Sprache unterschiedlich sein.

Mit dem Terminus Topik wird im Allgemeinen eine sprachliche Einheit bezeichnet, über die etwas in einer Äußerung ausgesagt wird (Reinhart 1981). Nur referentielle Ausdrücke können als Topik gelten. In vielen Sprachen wird das Topik durch eine referentielle Determiniererphrase (DP) ausgedrückt. Schwache (unbetonte) Pronomen und Klitika sind immer Topik. Die Sprachen können jedoch das Topik auch auf andere Art und Weise markieren, wie zum Beispiel durch die Topikalisierung des Elementes, durch seine Auslassung, durch die entsprechende Intonation oder Morphologie (vgl. Erteschik-Shir 2007). In den meisten Sprachen werden mehrere Mittel dafür gebraucht, um ein Element als Topik zu markieren.

Die Informationsstruktur als Schnittstelle zwischen Sprache und Kognition ist deshalb ein wichtiger Gegenstand der Untersuchungen im Rahmen der Linguistik, der Psycholinguistik und der Spracherwerbsforschung.

\section{ENTWICKLUNG DER INFORMATIONSSTRUKTUR}

Kinder, die ihre Erstsprache erwerben, haben im Laufe des Erwerbs der Informationsstruktur mehrere Aufgaben zu bewältigen. Sie müssen die linguistische Kompetenz für die entsprechende Verbindung vom Informationsstatus des Referenten mit den entsprechenden sprachlichen Ausdrücken und Strukturen erwerben. Darüber hinaus müssen sie die kognitive Fähigkeit erlernen, die eigene Perspektive von der Perspektive des Hörers zu unterscheiden. 
Studien im Rahmen der Spracherwerbsforschung zeigen, dass das grundlegende Wissen um die Informationsstruktur von den Kindern schon im Alter von ca. 2; 6 Jahren erworben wird (De Cat 2009). Kinder können schon in diesem Alter den Unterschied Topik vs. Fokus wahrnehmen. In diesem Alter entwickelt sich auch die linguistische Kompetenz, die für den Gebrauch der entsprechenden referentiellen Ausdrücke zuständig ist. Der Unterschied zwischen der definiten und indefiniten DP ist in den sprachlichen Daten der Kinder in diesem Alter zu sehen. Die Anzahl von Fehlern zeugt jedoch davon, dass Kinder in diesem Alter noch nicht vollständig das Zusammenspiel der Regeln des Strukturaufbaus mit den allgemeinen kognitiven Prozessen erlernt haben. Maratsos (1974) argumentiert, dass Kinder bis zum Alter von fünf Jahren Probleme mit der Wahrnehmung des Unterschieds zwischen der eigenen und der fremden Perspektive haben.

Ein wichtiger Teil der Sprachkompetenz, die mit der Informationsstruktur verbunden ist, ist der Gebrauch der Pronomen, die für die Referenzherstellung zuständig sind. Das referentielle Argument kann als Pronomen ausgedrückt werden, wenn es ein bekanntes Topik ist. Sowohl der Sprecher als auch der Hörer müssen das Argument als etabliertes Topik erkennen, wenn es als Pronomen ausgedrückt werden soll. Je prominenter das Argument im Diskurs ist, desto wahrscheinlicher ist der Gebrauch der Pronomen statt der Nominalphrase, um es auszudrücken. In einigen Sprachen besteht noch die Möglichkeit des Gebrauchs von Klitika (z.B. Französisch, Polnisch) oder von Nullargumenten (z.B. Deutsch, Polnisch).

Untersuchungen des Spracherwerbszeigen, dass Kinder den Gebrauch der Pronomen und der Klitika stufenweise erwerben. Jakubowicz u.a. (1998) belegen, dass Kinder, die Französisch als ihre Erstsprache (L1) erwerben, in derselben Zeit Klitika im Akkusativ auslassen und die Determinierer nicht, obwohl sie phonologisch dieselbe Form annehmen. In mehreren Studien wird festgelegt, dass Kinder dazu neigen, Pronomen zum Ausdruck der Subjekte häufiger als zu dem der Objekte zu benutzen (Pinker 1984; Bloom 1990; Hamann 1996). 2-3-jährige Kinder, die Englisch als L1 erwerben, gebrauchen Pronomen als Subjekte in ca. 51\% der Fälle und als Objekte in ca. $24 \%$ der Fälle (Bloom 1990). Bamberg (1987) untersucht den Gebrauch der Pronomen im L1-Erwerb des Deutschen und legt dabei auch fest, dass pronominale Formen zum Ausdruck des Subjekts bei 3-4 jährigen überwiegen.

Die Pronomen im Akkusativ werden von Kindern, die Deutsch als L1 erwerben, bei elizitierten Sprachuntersuchungen oft ausgelassen (Jakubowicz u.a. 1996; 1997; Ruigendijk u.a. 2010). Ruigendijk u.a. (2010) zeigen, dass 6-jährige Kinder, die L1 Deutsch erwerben, bei elizitierten Sprachdaten Pronomen zu 58\%, Determiniererphrasen zu 24\% und Nullargumente zu $10 \%$ zum Ausdruck des Objekts benutzen. Der Gebrauch der Pronomen für 
Akkusativobjekte wird in anderen Sprachen noch schneller von Kindern erworben. Ruigendijk u.a. (2010) bewiesen beispielsweise, dass Hebräisch erwerbende Kinder schon im Alter von zwei bis drei Jahren Pronomen in der Position des Objektes in ca. 70\% der Fälle gebrauchen.

Zusammenfassend kann man sagen, dass Kinder im Rahmen des Erstspracherwerbs den Gebrauch der Pronomen in verschiedenen Sprachen unterschiedlich schnell erwerben. Im Alter von ca. sieben bis acht Jahren sind die relevanten Hauptbereiche der linguistischen Kompetenz und die grundlegenden kognitiven Prozesse, die auf der Schnittstelle der Informationsstruktur zusammenspielen, bereits erworben. Das Zusammenspiel der Sprachstrukturaufbauregeln sowie der mentalen Repräsentation des Diskurses läuft schon in den Köpfen der Kinder in diesem Alter bereits relativ reibungslos ab.

\section{EMPIRISCHE UNTERSUCHUNG ZUM GEBRAUCH VON PRONOMEN BEI DEUTSCH ALS FRÜHE FREMDSPRACHE}

\subsection{Aufbau der Untersuchung}

Das Hauptanliegen der vorliegenden Studie ist die Erforschung der Entwicklung der Informationsstruktur beim frühen Erwerb des Deutschen als Fremdsprache (DaF). Die Untersuchung des Erwerbs von Sprachstrukturaufbauregeln und der mentalen Repräsentation des Diskurses bei Kindern, die im frühen Alter eine Fremdsprache erlernen, ist ein notwendiger Mosaikstein für das Bild des Zweitspracherwerbs, das trotz aller bisheriger Forschung noch bei weitem nicht scharf und vollständig genug ist.

Kinder, die älter als sechs Jahre alt sind, haben schon das Zusammenspiel von Regeln des Strukturaufbaus mit den allgemeinen kognitiven Prozessen in ihrer Erstsprache erworben. Sie können unterschiedliche Perspektiven des Sprechers und des Hörers wahrnehmen. Sie sind im Stande, die mentale Repräsentation des Diskurses aufzubauen und die dem informationellen Status des Referenten entsprechenden Ausdrücke für Argumente zu wählen sowie eine sprachliche Struktur aufzubauen, die die relevanten Verhältnisse der Informationsstruktur berücksichtigt. Kurzum - die Informationsstruktur, d.h. die Schnittstelle zwischen der Struktur und der Bedeutung von sprachlichen Äußerungen, ist in der Verarbeitung in ihrer Erstsprache zu diesem Zeitpunkt wirksam. Der Einsatz der Fähigkeit, die mentale Repräsentation des Diskurses aufzubauen, muss jedoch in der Fremdsprache nicht automatisch erfolgen. Die allgemeinkognitiven Elemente des Zusammenspiels sind zweifellos in den Köpfen der Lerner da. Man kann anneh- 
men, dass Kinder die unterschiedlichen Perspektiven des Sprechers und des Hörers auch in der Fremdsprache wahrnehmen können, wenn sie es schon in der L1 erlernt haben. Die anderen Elemente sind jedoch mit der linguistischen Kompetenz verbunden, die für den Gebrauch der entsprechenden referentiellen Ausdrücke zuständig ist. Sie sind spezifisch für jede Sprache und müssen von Kindern in der Fremdsprache erst erworben werden. Der Aufbau der mentalen Repräsentation des Diskurses kann auch teilweise sprachspezifisch sein und die Fähigkeit, die Informationsstruktur des Gesprächs in einer Fremdsprache aufzubauen, muss auch von Kindern neu geübt werden.

Die vorliegende Untersuchung hat zum Ziel, einen Einblick in die Dynamik der Entwicklung der Informationsstruktur in der früh erworbenen Fremdsprache durch die Erforschung des Gebrauchs der Pronomen der 3. Person als Subjekt und Objekt bei Kindern zu liefern, die Deutsch als Fremdsprache erwerben. Wie bereits oben erklärt ist die Benutzung der Pronomen für die Herstellung der Referenz zuständig und dadurch mit den wichtigsten Phänomenen verbunden, die an der Schnittstelle der Informationsstruktur eine Rolle spielen.

Bei der vorliegenden Studie handelt es sich um eine Longitudinaluntersuchung von Daten von Kindern, deren L1 Polnisch ist, und die Deutsch als ihre erste frühe Fremdsprache unter schulischen Bedingungen erlernen. Die Untersuchungsgruppe bilden 13 Kinder, die den Erwerb des Deutschen als Fremdsprache mit sieben oder neun Jahren begonnen haben. Die erste Gruppe umfasst Kinder, die zu Beginn der Erhebung im Alter von ca. zehn Jahren waren und in der zweiten Gruppe sind Kinder, die im Alter von ca. elf Jahren waren. Die Kinder der ersten Gruppe hatten zu Beginn der Erhebung 30 Kontaktmonate $(\mathrm{KM})$ zu Deutsch und die der zweiten 40 Kontaktmonate hinter sich. Die dritte Gruppe umfasst Schüler, die mit ca. neun Jahren begonnen haben, Deutsch als Fremdsprache zu erlernen. Im Erhebungszeitraum waren die Kinder der dritten Gruppe durchschnittlich zwölf Jahre alt. Zu Beginn der Aufnahmen hatten sie 30 Kontaktmonate hinter sich. Die Angaben zu den Schülergruppen werden in der Tabelle 1 zusammengefasst:

Tabelle 1. Übersicht über die Datenbasis

\begin{tabular}{|c|c|c|c|c|}
\hline Gruppe & $\begin{array}{c}\text { Anzahl } \\
\text { der Kinder }\end{array}$ & $\begin{array}{c}\text { Alter bei } \\
\text { Erwerbsbeginn }\end{array}$ & $\begin{array}{c}\text { Alter zu Beginn } \\
\text { der Erhebung }\end{array}$ & $\begin{array}{c}\text { KM zu Beginn } \\
\text { der Erhebung }\end{array}$ \\
\hline 1 & 4 & ca. 7 & ca. 10 & 30 \\
\hline 2 & 3 & ca. 7 & ca. 11 & 40 \\
\hline 3 & 6 & ca. 9 & ca. 12 & 30 \\
\hline
\end{tabular}


Alle Aufnahmen dauerten ca. eine Stunde und wurden einmal im Monat durchgeführt. Die Gruppen wurden während der Interaktion miteinander und mit dem Interviewer aufgenommen. Es handelte sich dabei um Spontansprachdaten, die bei schwach vorstrukturierten Spielen gewonnen wurden, die geeignete Sprechanlässe für Kinder in diesem Alter lieferten.

Die Kinder erhielten wöchentlich zwei bis drei Unterrichtsstunden Deutsch als Fremdsprache. Im Unterricht wurden verschiedene Lehrmaterialien im Rahmen deskommunikativen Ansatzes in der Didaktik eingesetzt. Die Unterrichtsbeobachtung hat auch gezeigt, dass die Kinder nach den Grundprinzipien des kommunikativen Ansatzes in der Fremdsprachendidaktik unterrichtet worden sind.

\subsection{Ergebnisse der Untersuchung}

In allen drei Gruppen wurden alle Subjekt- und Objektrealisierungen in der 3. Person untersucht. Für die Untersuchung der Ausdrücke, die Kinder für das Akkusativobjekt benutzt haben, wurden transitive Kontexte berücksichtigt. Unter den untersuchten Kontexten befinden sich sowohl Kontexte, in denen das Subjekt oder das Objekt das bekannte Topik ist, als auch solche, in denen das Subjekt oder das Objekt nicht das bekannte Topik ist. Nach Givon (1984: 169-171) werden das Subjekt als primäres bekanntes Topik und das Objekt als sekundäres bekanntes Topik in den Kontexten betrachtet, in denen ein anderes Element, beispielsweise das Verb, im Fokus steht, und das Subjekt und das Objekt schon bekannte Informationen im Diskurs wiedergeben. In Spontandaten kann man die Topik- von den Nicht-Topik-Kontexten schwierig voneinander unterscheiden und die Forscher haben verschiedene Kriterien für die Unterscheidung vorgeschlagen (siehe beispielsweise Pirvulescu 2006). Im natürlichen Gebrauch einer Sprache sind jedoch jene Situationen relativ häufig, in denen das Subjekt oder das Akkusativobjekt das bekannte Topik ist. In Spontandaten kann man daher davon ausgehen, dass ein gewisser Anteil von den Kontexten ermöglicht, das Subjekt oder das Objekt als Pronomen oder als Nullargument auszudrücken. In der Auswertung der Daten wird nicht darauf Rücksicht genommen, ob der Gebrauch der Pronomen aus pragmatischen Gründen angebracht ist oder ob die Form der Pronomen morphologisch richtig ist.

Der Gebrauch der referentiellen Ausdrücke, also der Nominalphrasen (NP), der Pronomen und der Nullargumente (Null) als Subjekte oder als Objekt ein den einzelnen Monaten in der ersten, zweiten und dritten Gruppe wird entsprechend in den Tabellen 2, 3 und 4 dargestellt. 
Tabelle 2. Subjekt- und Objektrealisierung pro Monat - Gruppe 1

\begin{tabular}{|l|c|r|r|r|r|r|r|}
\hline Gruppe 1 & $32 \mathrm{KM}$ & $33 \mathrm{KM}$ & $34 \mathrm{KM}$ & $35 \mathrm{KM}$ & $36 \mathrm{KM}$ & $37 \mathrm{KM}$ & $38 \mathrm{KM}$ \\
\hline \multicolumn{7}{|c|}{ Subjektrealisierung } \\
\hline volle NP & $44 \%$ & $80 \%$ & $82 \%$ & $100 \%$ & - & $9 \%$ & $0 \%$ \\
\hline Pronomen & $33 \%$ & $20 \%$ & $18 \%$ & $0 \%$ & - & $89 \%$ & $89 \%$ \\
\hline Null & $22 \%$ & $0 \%$ & $0 \%$ & $0 \%$ & - & $2 \%$ & $11 \%$ \\
\hline \multicolumn{8}{|c|}{ Objektrealisierung } \\
\hline volle NP & $100 \%$ & $75 \%$ & $83 \%$ & $96 \%$ & $94 \%$ & $50 \%$ & - \\
\hline Pronomen & $0 \%$ & $0 \%$ & $8 \%$ & $0 \%$ & $0 \%$ & $50 \%$ & - \\
\hline Null & $0 \%$ & $25 \%$ & $8 \%$ & $4 \%$ & $6 \%$ & $0 \%$ & - \\
\hline
\end{tabular}

Tabelle 3. Subjekt- und Objektrealisierung pro Monat - Gruppe 2

\begin{tabular}{|l|r|r|r|r|r|r|r|r|}
\hline Gruppe 2 & $41 \mathrm{KM}$ & $42 \mathrm{KM}$ & $43 \mathrm{KM}$ & $44 \mathrm{KM}$ & $45 \mathrm{KM}$ & $46 \mathrm{KM}$ & $47 \mathrm{KM}$ & $48 \mathrm{KM}$ \\
\hline \multicolumn{8}{|c|}{ Subjektrealisierung } \\
\hline volle NP & $100 \%$ & $95 \%$ & $100 \%$ & $94 \%$ & $93 \%$ & $89 \%$ & $85 \%$ & $43 \%$ \\
\hline Pronomen & $0 \%$ & $0 \%$ & $0 \%$ & $0 \%$ & $0 \%$ & $0 \%$ & $15 \%$ & $57 \%$ \\
\hline Null & $0 \%$ & $5 \%$ & $0 \%$ & $5 \%$ & $7 \%$ & $11 \%$ & $0 \%$ & $0 \%$ \\
\hline \multicolumn{8}{|c|}{ Objektrealisierung } \\
\hline volle NP & $50 \%$ & $90 \%$ & $97 \%$ & $100 \%$ & $99 \%$ & $94 \%$ & $50 \%$ & $100 \%$ \\
\hline Pronomen & $50 \%$ & $0 \%$ & $1 \%$ & $0 \%$ & $0 \%$ & $5 \%$ & $50 \%$ & $0 \%$ \\
\hline Null & $0 \%$ & $10 \%$ & $2 \%$ & $0 \%$ & $1 \%$ & $1 \%$ & $0 \%$ & $0 \%$ \\
\hline
\end{tabular}

Tabelle 4. Subjekt- und Objektrealisierung pro Monat - Gruppe 3

\begin{tabular}{|l|r|r|r|r|r|r|r|}
\hline Gruppe 3 & $32 \mathrm{KM}$ & $33 \mathrm{KM}$ & $34 \mathrm{KM}$ & $35 \mathrm{KM}$ & $36 \mathrm{KM}$ & $37 \mathrm{KM}$ & $38 \mathrm{KM}$ \\
\hline \multicolumn{7}{|c|}{ Subjektrealisierung } \\
\hline volle NP & $0 \%$ & $50 \%$ & $91 \%$ & $100 \%$ & $100 \%$ & $29 \%$ & $46 \%$ \\
\hline Pronomen & $67 \%$ & $17 \%$ & $5 \%$ & $0 \%$ & $0 \%$ & $49 \%$ & $54 \%$ \\
\hline Null & $33 \%$ & $33 \%$ & $5 \%$ & $0 \%$ & $0 \%$ & $22 \%$ & $0 \%$ \\
\hline \multicolumn{7}{|c|}{ Objektrealisierung } \\
\hline volle NP & $100 \%$ & $85 \%$ & $100 \%$ & $95 \%$ & $98 \%$ & $83 \%$ & $100 \%$ \\
\hline Pronomen & $0 \%$ & $3 \%$ & $0 \%$ & $2 \%$ & $1 \%$ & $8 \%$ & $0 \%$ \\
\hline Null & $0 \%$ & $13 \%$ & $0 \%$ & $3 \%$ & $1 \%$ & $8 \%$ & $0 \%$ \\
\hline
\end{tabular}

Den Tabellen 2-4 kann man entnehmen, dass die Subjektrealisierung in verschiedenen Monaten in allen Gruppen sehr unterschiedlich ist. In einigen Aufnahmen ist der Anteil der Pronomen an der Gesamtanzahl der Subjekte 
in der 3. Person sehr hoch, z.B. Tabelle 2 - Gruppe 1: $37 \mathrm{KM}$ und 38 KM; Tabelle 3 - Gruppe 2: 48 KM; Tabelle 4 - Gruppe 3: 32 KM, 38 KM. Dieser Befund kann teilweise auf die unterschiedlichen Sprachanstöße in verschiedenen Monaten zurückgeführt werden. Festzuhalten ist jedoch bei der Untersuchung der Subjektrealisierung, dass alle Gruppen Pronomen zum Ausdruck des Subjekts gebrauchen können.

Eine Äußerung eines Kindes, die ein Pronomen zum Ausdruck des Subjektes enthält, ist im Beispiel 1 aus den Daten der Gruppe 3 zu sehen:

1. Person A: Ania geht ins Schwimmbad

Person B: Sie geht ins Schwimmbad nicht.

An diesem Beispiel sieht man, dass Kinder die Pronomen in der richtigen morphologischen Form und im richtigen pragmatischen Kontext einsetzen können.

Wie die Tabellen 2-4 zeigen, sieht der Gebrauch der referentiellen Ausdrücke im Falle des Objektes anders als im Falle des Subjektes aus. In allen Monaten und in allen Gruppen wird meistens die NP zum Ausdruck des Akkusativobjekts gewählt. In einigen Monaten werden gar keine Pronomen von Kindern in den transitiven Kontexten benutzt (siehe Tabelle 2 - Gruppe 1: $32 \mathrm{KM}, 33 \mathrm{KM}, 35 \mathrm{KM}, 36 \mathrm{KM}$; Tabelle 3 - Gruppe 2: $42 \mathrm{KM}, 44 \mathrm{KM}$, $45 \mathrm{KM}, 48 \mathrm{KM}$; Tabelle 4 - Gruppe 3: $32 \mathrm{KM}, 34 \mathrm{KM}, 38 \mathrm{KM})$. In anderen Monaten ist der Anteil der Pronomen an allen Ausdrücken, die für das Akkusativobjekt benutzt worden sind, nicht hoch. Nur in wenigen Monaten liegt der Wert bei 50\% (siehe Tabelle 2 - Gruppe 1: 37 KM, Tabelle 3 - Gruppe 2: $41 \mathrm{KM}$ und $47 \mathrm{KM}$ ).

Tabelle 5. Subjekt- und Objektrealisierung in allen Aufnahmen: Gesamt- und Prozentzahlen Gruppe 1, 2 und 3

\begin{tabular}{|l|c|c|c|c|c|c|}
\hline \multirow{2}{*}{ Ausdruck } & \multicolumn{2}{|c|}{ Gruppe 1 } & \multicolumn{2}{c|}{ Gruppe 2 } & \multicolumn{2}{c|}{ Gruppe 3 } \\
\cline { 2 - 7 } & Gesamt & $\begin{array}{c}\text { Gesamt } \\
\text { Prozent }\end{array}$ & Gesamt & $\begin{array}{c}\text { Gesamt } \\
\text { Prozent }\end{array}$ & Gesamt & $\begin{array}{c}\text { Gesamt } \\
\text { Prozent }\end{array}$ \\
\hline \multicolumn{7}{|c|}{ Subjektrealisierung } \\
\hline volle NP & 48 & $38 \%$ & 202 & $88 \%$ & 138 & $64 \%$ \\
\hline Pronomen & 73 & $58 \%$ & 16 & $7 \%$ & 64 & $29 \%$ \\
\hline Null & 4 & $3 \%$ & 9 & $4 \%$ & 15 & $7 \%$ \\
\hline \multicolumn{7}{|c|}{ Objektrealisierung } \\
\hline volle NP & 192 & $91 \%$ & 351 & $95 \%$ & 327 & $95 \%$ \\
\hline Pronomen & 10 & $5 \%$ & 13 & $4 \%$ & 6 & $2 \%$ \\
\hline Null & 10 & $5 \%$ & 7 & $2 \%$ & 10 & $3 \%$ \\
\hline
\end{tabular}


In der Tabelle 5 werden die Gesamtdaten zur Realisierung der Subjekte und Objekte der dritten Person in allen Aufnahmen für die Gruppe 1, 2 und 3 dargestellt. Die Tabellen beinhalten sowohl Gesamt- als auch Prozentzahlen.

Der Tabelle 5 kann man entnehmen, dass der Gebrauch der Pronomen in allen Gruppen im Falle der Subjekte höher als im Falle der Objekte liegt. Die erste Gruppe gebraucht Pronomen als Subjekte ziemlich häufig (58\%), die zweite seltener $(7 \%)$ und die dritte wiederum häufiger (29\%). Im Vergleich dazu ist der Gebrauch der Pronomen für Akkusativobjekte ein relativ seltenes Phänomen. Die Kinder aus Gruppe 1 haben das Pronomen insgesamt in $5 \%$, aus Gruppe 2 in $4 \%$ und aus Gruppe 3 in 2\% der transitiven Kontexte benutzt (siehe Tabelle 5). Dies ist zweifellos viel weniger als es im normalen Redefluss in einer natürlichen Sprache der Fall ist.

Wenn man die Subjektrealisierung der DaF-Kinder mit den L1-Kindern vergleicht, dann ist kein großer Unterschied festzustellen. Die Werte für den Gebrauch der Pronomen als Akkusativobjekte liegen aber viel niedriger als jene Prozentzahlen, die in Studien zum Erwerb des Deutschen als Erstsprache genannt werden. Ruigendijk u.a. (2010) haben den Wert von 58\% für Pronomen in elizitierten Sprachdaten von 6-jährigen L1-Kindern genannt. In experimentellen Studien sind die Werte noch höher. Bei Varlokosta u.a. (2016) haben 5-jährige Kinder, die Deutsch als L1 erwerben, in ihrem Experiment ein Pronomen für das Akkusativobjekt in 89\% der Fälle benutzt. Die Anzahl der Pronomen bei den Kindern war nur 4\% niedriger als ihre Anzahl in den Daten der Erwachsenen. Die Prozentwerte in den spontanen Sprachdaten und in den experimentellen Daten können natürlich nicht direkt miteinander verglichen werden. Vor dem Hintergrund der L1-Daten sieht man jedoch deutlich, dass der Gebrauch der Pronomen zum Ausdruck des Akkusativobjektes in der frühen Fremdsprache gering ist.

\section{SCHLUSSFOLGERUNGEN}

Zusammenfassend kann man sagen, dass der Gebrauch der Pronomen im Verlauf des frühen Erwerbs des Deutschen als Fremdsprache bei Kindern, bei denen der Erwerb im Alter von sieben bis neun Jahren begonnen hat, auf eine relativ langsame aber stabile Entwicklung der Informationsstruktur hindeutet. Die Kinder benutzen die Pronomen zum Ausdruck des Subjektes relativ oft, was davon zeugen kann, dass sie die schon in ihrer Erstsprache erworbene Fähigkeit, die mentale Repräsentation des Diskurses aufzubauen, und die Perspektiven des Sprechers sowie des Hörers wahrzunehmen, in ihrer frühen Fremdsprache einsetzen können. Wenn man die Ergebnisse des Gebrauchs der Pronomen zum Ausdruck des Akkusativobjektes mitberücksich- 
tigt, dann sieht man einen deutlichen Unterschied zwischen der Dynamik der Entwicklung der frühen Fremdsprache und der Erstsprache. Die DaF-Kinder benutzen nur selten die Pronomen zum Ausdruck des Objektes im Gegensatz zu L1-Kindern und zum natürlichen Gebrauch des Deutschen bei Erwachsenen. Zum Teil kann dieser Befund mit dem späteren Erlernen der Formen der Pronomen für den Akkusativ im Vergleich zu den Formen für den Nominativ erklärt werden. Da die Kinder jedoch im Rahmen des Unterrichts schon das ganze Pronomensystem kennengelernt haben, können die Probleme mit den Akkusativformen den Unterschied im Gebrauch des Pronomens bei Subjekt vs. Objekt nicht vollständig erklären.

Das Ergebnis der vorliegenden Untersuchung, d.h. der relativ häufige Gebrauch der Pronomen zum Ausdruck des Subjektes und die seltene Benutzung der Pronomen zum Ausdruck des Akkusativobjektes in der frühen Fremdsprache, kann auf die noch nicht reibungslos funktionierende Informationsstruktur in der frühen Fremdsprache bei Kindern zurückgeführt werden. Im Rahmen der Sprachverarbeitung ist die Interpretation der Referenz der Subjekte ein grundlegender Prozess, wenn das Subjekt das primäre bekannte Topik ist. Die Interpretation der Referenz der Objekte, die das sekundäre bekannte Topik sein können, ist ein Prozess, der einen zusätzlichen Verarbeitungsaufwand benötigt. Die Bindung des Pronomens in der Position des Subjektes an das entsprechende Element in der sich entwickelnden mentalen Repräsentation des Diskurses in der frühen Fremdsprache ist also ein schnellerer Prozess als es im Falle des Objektes ist.

Im Einklang mit Pfeiffer (2001a; 2001b) wird in der vorliegenden Studie davon ausgegangen, dass die wichtigste Aufgabe der Glottodidaktik darin besteht, fremdsprachliche Lehr- und Lernprozesse $\mathrm{zu}$ beobachten, $\mathrm{zu}$ beschreiben, zu analysieren und zu erklären. Im Rahmen der Untersuchung wurde der Versuch unternommen, einen Einblick in die mentalen Prozesse zu gewinnen, die im Kopf der Lerner des Deutschen als frühe Fremdsprache verlaufen. Nur dann, wenn man mehr Wissen um die mentalen Prozesse bei den Lernern hat, kann man wissenschaftlich fundierte Hinweise für die Gestaltung der anderen Elemente des glottodidaktischen Gefüge vorschlagen. Die in der vorliegenden Untersuchung erzielten Erkenntnisse weisen darauf hin, dass der Entwicklung der Informationsstruktur bei den Lernern mehr Aufmerksamkeit im Rahmen des frühen Fremdsprachunterrichts geschenkt werden soll.

\section{LITERATURVERZEICHNIS}

Bamberg, M. (1987). The acquisition of narratives. Learning to use language. Berlin, New York, Amsterdam: Mouton de Gruyter. 
Bielicka, M. (2014). Der sprachliche Hintergrund von Kindern in zweisprachigen Kindergärten mit den Arbeitssprachen Polnisch und Deutsch - eine vergleichende Studie. Zur Relevanz von Forschungsergebnissen aus dem Bereich der Immersionsmethode für DaF-Studierende und DaF-Lehrkräfte. In: S. Adamczak-Krysztofowicz / A. Szczepaniak-Kozak (Hrsg.), Kultur Kommunikation - Kreativität - Reflexivität (S. 207-217). Frankfurt a.M.: Peter Lang.

Bloom, P. (1990). Subjectless Sentences in Child Language. LinguisticInquiry, 21 (4), 491-504.

Błażek, A. (2007). Möglichkeiten und Grenzen der Messung interkultureller Kompetenz. Glottodidactica. An International Journal of Applied Linguistics, 33, 71-82.

De Cat, C. (2009). Experimental evidence for preschoolers' mastery of "topic". Language Acquisition, 16, 224-239.

Długosz, K. (2017a). Die Relation zwischen kognitiven Faktoren und Code-Switching im Fremdsprachenunterricht. Psycho- und neurolinguistische Dimensionen. Studia Niemcoznawcze, 59, 695-709.

Długosz, K. (2017b). Zum Einfluss von soziopragmatischen Faktoren auf Code-Switching. In: B. Mikołajczyk / J. Taborek / M. Woźniak / M. Woźniacka / W. Zabrocki (Hrsg.), Jezzyk w Poznaniu 8 (S. 9-15). Poznań: Wydawnictwo Rys.

Erteschik-Shir, N. (2007). Information structure. Oxford: Oxford University Press.

Givon, T. (1984). Syntax. A Functional-Typological Introduction. Volume 1. Amsterdam: Benjamins.

Grucza, F. (1978). Teoria komunikacji jezzykowej a glottodydaktyka. Warszawa: Wydawnictwa UW.

Grucza, F. (1983). Zum Gegenstand und zur inneren Gliederung der Linguistik und der Glottodidaktik. Kwartalnik Neofilologiczny, XXX, 217-234.

Grucza, F. (1993). Ansätze zu einer Theorie der Ausbildung von Fremdsprachenlehrern. In: F. Grucza / H.-J. Krumm / B. Grucza (Hrsg.), Beiträge zur wissenschaftlichen Fundierung der Ausbildung von Fremdsprachenlehrern (S. 7-96). Warszawa: Wydawnictwa UW.

Grucza, F. (2007). Lingwistyka stosowana. Historia - Zadania - Osiagnięcia. Warszawa: Euro-Edukacja.

Hamann, C. (1996). Null Arguments in German Child Language. Language Acquisition, 5, 155-208.

Jakubowicz, C. / Müller, N. / Ok-Kyung, K. / Riemer, B. / Rigaut, C. (1996). On the acquisition of the pronominal system in French and German. In: A. Springfellow / D. CahanaAmitay / E. Hughes / A. Zukowski (eds.), Proceedings of the 20th Annual Boston University Conference on Language Development (pp. 374-385). Somerville, MA: Cascadilla Press.

Jakubowicz, C. / Müller, N. / Riemer, B. / Rigaut, C. (1997). The case of subject and object omissions in French and German. In: E. Hughes / M. Hughes / A. Greenhill (eds.), Proceedings of the 21 Annual Boston University Conference on Language Development (pp. 331-342). Somerville, MA: Cascadilla Press.

Jakubowicz, C. / Nash, L. / Rigaut, C. / Gerard, Ch.-L. (1998). Determiners and pronouns in French-speaking children with SLI. Language Acquisition, 7, 113-160.

Lambrecht, K. (1994). Information structure and sentence form: Topic, focus and the mental representations of discourse referents. Cambridge, UK: Cambridge Univ. Press. DOI: 10.1017/ CBO9780511620607

Maratsos, M. (1974). Preschool children's use of definite and indefinite articles. Child Development, 45, 446-455.

Pfeiffer, W. (1980). Kierunki badań glottodydaktyki. In: M. Szczodrowski (Hrsg.), Efektywność nauczania języków obcych na kursach pozaszkolnych (S. 7-27). Szczecin: Towarzystwo Wiedzy Powszechnej.

Pfeiffer, W. (1995). Zur Krise der wissenschaftlichen Fremdsprachendidaktik. Ein methodologischer Essay. In: H. Popp (Hrsg.), Deutsch als Fremdsprache. An den Quellen eines Faches (S. 637-647). Berlin: Iudicium. 
Pfeiffer, W. (2001a). Glottodidaktik als Wissenschaft. In: K. Aguado / C. Riemer (Hrsg.), Wege und Ziele. Zur Theorie, Empirie und Praxis des Deutschen als Fremdsprache (und anderer Fremdsprachen). Festschrift für Gert Henrici zum 60. Geburtstag (S. 63-96). Hohengehren: Schneider Verlag.

Pfeiffer, W. (2001b). Nauka języków obcych. Od praktyki do praktyki. Poznań: Wagros.

Pinker, S. (1984). Language Learnability and Language Development. Cambridge, Massachusetts: Harvard University Press.

Pirvulescu, M. (2006). Theoretical implications of clitic omissions in early French: Spontaneous vs. elicited production. Catalan Journal of Linguistics, 5, 221-236.

Reinhart, T. (1981). Pragmatics and linguistics: an analysis of sentence topics. Philosophica, 27, 53-94.

Ruigendijk, E. / Friedmann N. / Novogrodsky R. / Balaban, N. (2010). Symmetry in comprehension and production of pronouns: A comparison of German and Hebrew. Lingua, 120, 1991-2005.

Sadownik, B. (1997). Glottodidaktische und psycholinguistische Aspekte des Fremdsprachenerwerbs: Lernerperspektive. Lublin: Wydawnictwo UMCS.

Skowronek, B. (2013). Glottodidaktik und Fremdsprachenunterricht in der Diskussion. Poznań: Wydawnictwo Naukowe UAM.

Sopata, A. (2004). Universalgrammatik und Fremdsprachendidaktik. Danziger Beiträge zur Germanistik. Band 14. Frankfurt am Main: Peter Lang Verlag.

Sopata, A. (2010). Der frühe Fremdsprachenunterricht - je früher desto besser? Glottodidactica. An International Journal of Applied Linguistics, 36, 95-106.

Sopata, A. (2017). Interaktion zwischen pragmatischen und syntaktischen Aspekten im Erwerb des Deutschen als Fremdsprache. In: J. Zhu / J. Zhao / M. Szurawitzki (Hrsg.), Akten des XIII. Internationalen Germanistenkongresses Shanghai 2015 - Germanistik zwischen Tradition und Innovation (S. 51-62). Frankfurt am Main: Peter Lang.

Varlokosta, S. / Belletti, A. / Costa, J. / Friedmann, N. / Gavarró, A. / Grohmann, K. / Guasti, M. / Tuller, L. / Lobo, M. / Anđelković, D. / Argemí, N. / Avram, L. / Berends, S. / Brunetto, V. / Delage, H. / Ezeizabarrena, M. / Fattal, I. / Haman, E. / van Hout, A. / Jensen de López, K. / Katsos, N. / Kologranic, L. / Krstić, N. / Kuvac Kraljevic, J. / Miękisz, A. / Nerantzini, M. / Queraltó, C. / Radic, Z. / Ruiz, S. / Sauerland, U. / Sevcenco, A. / Smoczyńska, M. / Theodorou, E. / van der Lely, H. / Veenstra, A. / Weston, J. / Yachini, M. / Yatsushiro, K. (2016). A cross-linguistic study of the acquisition of clitic and pronoun production, Language Acquisition, 23, 1-26. DOI: 10.1080/10489223.2015.1028628.

Zimmermann, M. (2016). Information Structure. In: M. Aronoff (ed.), Oxford Bibliographies in Linguistics. New York: Oxford University Press. DOI: 10.1093/obo/9780199772810-0130.

Zuzok, N. (2010). Interkulturelle Kompetenz im Fremdsprachenunterricht Deutsch nach Englisch. Glottodidactica. An International Journal of Applied Linguistics, 36, 235-243.

Received: 09.08.2018; revised: 30.08.2018 